\title{
Solo sódico tratado com gesso agrícola, composto de lixo urbano e vinhaça ${ }^{1}$
}

Maria de F. G. dos Santos ${ }^{2}$ Francisco A. de Oliveira ${ }^{3}$ Lourival F. Cavalcante ${ }^{3}$, José F. de Medeiros ${ }^{4} \&$ Carlos C. de Souza ${ }^{5}$

\footnotetext{
${ }^{1}$ Parte da Dissertação de Mestrado do primeiro autor, apresentada na UFPB, trabalho realizado com apoio financeiro do CNPq (Programa Nordeste de Pesquisa e Pós-Graduação)

${ }^{2}$ UFPB. Rua Juliana Taveira 26, CEP 58135-000, Esperança, PB. Fone: (83) 3361-2625 (Foto)

${ }^{3}$ Depto de Solos e Engenharia Rural, CCA/UFPB, CEP 58397-000, Areia, PB. Fone:(83) 3362-2300, R.251. E-mail: oliveira.@cca.ufpb.br; lofeca@cca.ufpb.br

${ }^{4}$ ESAM, ENA, CP 137, CEP 59625-900, Mossoró, RN. Fone: (84) 321-2100. E-mail: jfmedeir@esam.br

${ }^{6}$ CCA/UFPB, CEP 58397-000, Areia, PB. Fone: (83) 535-2662. E-mail: ccleide@cca.ufpb.br
}

Protocolo 198 - 15/12/2003 - Aprovado em 20/1/2005

\begin{abstract}
Resumo: O manejo inadequado da água de irrigação tem causado problemas de salinidade e sodicidade do solo nos perímetros irrigados com redução significativa na produtividade das culturas. Objetivando-se avaliar os efeitos da necessidade do gesso (NG): 0 e $40 \%$, do composto de lixo urbano (CLU): 0 e $20 \mathrm{t} \mathrm{ha}^{-1}$ e do período de incubação com vinhaça: 15, 30, 45 e 60 dias, sobre a recuperação de um solo sódico do projeto de irrigação São Gonçalo, no Estado da Paraíba, conduziu-se um experimento em casa-de-vegetação, do CCA/UFPB. Usou-se o delineamento em blocos ao acaso em esquema fatorial: $2 \times 2 \times 4$, com três repetições e o algodoeiro herbáceo, cultivar CNPA-7H, como cultura teste. O gesso + CLU aumentou a percentagem de emergência (\%E), a biomassa seca (BS) e o $\mathrm{Ca}^{2+}$ e diminuiu os teores de $\mathrm{Na}^{+}$e $\circ \mathrm{pH}$ nos lixiviados, em relação ao solo incubado com vinhaça, enquanto o $\mathrm{Ca}^{2+}$ aumentou com a NG mas diminuiu com - CLU. Para os períodos de incubação ocorreu redução linear nos teores de $\mathrm{Na}^{+}$na presença da NG e do CLU, isolados, e quando associados constatou-se redução também do $\mathrm{Ca}^{2+}$ e da condutividade elétrica (CE) em efeitos quadráticos e aumento do $\mathrm{pH}$. A incubação do solo apenas com vinhaça concorreu para aumentar a \%E e a $\mathrm{CE}$ em efeitos quadráticos e reduzir, de forma linear, a BS e o $\mathrm{Ca}^{2+}$.
\end{abstract}

Palavras-chave: sódio trocável, manejo do solo, recuperação

\section{Sodic soil treated with agricultural gypsum, urban waste compost and vinasse}

\begin{abstract}
The inadequate management of the irrigation water have caused salinity and sodicity problems in the irrigated perimeters with significant reduction of crop yields. This research was carried out in a greenhouse of CCA/UFPB, with the objective of studying the effects of necessity of the gypsum (NG): 0 and 40\%, urban waste compost (CLU): 0 and $20 \mathrm{t} \mathrm{ha}^{-1}$ and periods of incubation with vinasse: 15, 30, 45 and 60 days, on the reclamation of a sodic soil, from the "São Gonçalo Irrigation Project", in the State of Paraiba, Brazil. The experiment was conducted in randomized block design in a $2 \times 2 \times 4$ factorial scheme, with three repetitions and herbaceous cotton variety CNPA-7H as test crop. The treatments of gypsum + CLU increased the emergence percentage $(\% \mathrm{E})$, the dry matter $(\mathrm{DM})$ and $\mathrm{Ca}^{2+}$ and decreased the levels of $\mathrm{Na}^{+}$and $\mathrm{pH}$ of the leached solution, in relation to the soil incubated with vinasse, while $\mathrm{Ca}^{2+}$, increased with gypsum and decreased with CLU. For the incubation periods, there was linear reduction in $\mathrm{Na}^{+}$contents in the presence of NG and CLU, isolated and when associated, there was also decrease of $\mathrm{Ca}^{2+}$ and of the electrical conductivity (CE) presenting quadratic effects and increase of the $\mathrm{pH}$. The incubation periods only with vinasse increased the $\% \mathrm{E}$ and the $\mathrm{CE}$ showing quadratic effect and reduced linearly, form the DM and the $\mathrm{Ca}^{2+}$.
\end{abstract}

Key words: exchangeable sodium, soil management, reclamation 


\section{INTRODUÇÃO}

A crescente demanda por alimentos tornou o uso da irrigação imperativo em todo o mundo, sobretudo em regiões semi-áridas, a exemplo do Nordeste brasileiro, onde a ocorrência de déficits hídricos tem limitado o rendimento das culturas na maior parte do ano. Para Christofidis (2001), da área total mundial cultivada (cerca de 1,51 bilhão de hectares), 17,7\% são explorados sob irrigação e respondem por $40 \%$ do total das colheitas agrícolas; apenas 7,7\% do total dos 38,3 milhões de hectares cultivados no Brasil são irrigados, mas contribuem com $35 \%$ da produção de alimentos. Nessas áreas, o manejo inadequado da irrigação tem contribuído sobremaneira para o surgimento de problemas relacionados com a salinidade e/ou sodicidade no mundo inteiro, com decréscimos significativos nos rendimentos das culturas, causando sérios prejuízos ambientais e sociais levando ao abandono, em certos casos, expressivas áreas nos perímetros irrigados. Estima-se que cerca de $50 \%$ das áreas irrigadas do mundo estejam afetadas por sais ou por problemas associados e que, anualmente, são abandonados em torno de 10 milhões de hectares devido a esses entraves (Rhoades \& Loveday, 1990).

Os problemas advindos da salinidade e/ou sodicidade são mais restritos aos solos irrigados das regiões áridas e semiáridas, onde têm afetado cerca de $33 \%$ da área potencialmente arável no mundo (Gupta \& Abrol, 1990). Cerca de 52\% do Nordeste brasileiro são constituídos de clima semi-árido (SUDENE, 1977), em que em vários perímetros aí irrigados o problema tem limitado seriamente a produção agrícola (Damasceno, 1978; Góes, 1978; Cordeiro \& Millar, 1978; Cordeiro et al., 1988; Bernardo, 1995); em casos extremos, áreas marginais improdutivas afetadas por sais e/ou sódio trocável têm sido abandonadas (Valdivieso, 1986)

O acúmulo progressivo de sais solúveis e/ou sódio trocável tem afetado desfavoravelmente certos atributos do solo, tais como: $\mathrm{pH}$, condutividade elétrica, complexo sortivo, sódio trocável, floculação do complexo argila-húmus, retenção de umidade e permeabilidade, com efeitos deletérios no crescimento e no rendimento das culturas, bem como na biomassa microbiana (Santos, 1997). Quando se trata genericamente de solos salinos, a recuperação da área pode ser feita pela lavagem da camada do solo explorada pelo sistema radicular para lixiviar os sais solúveis para camadas mais profundas do solo, ou para fora da área de cultivo mas, quando o problema diz respeito ao sódio trocável, para a recuperação dessas áreas torna-se indispensável a utilização de corretivos para neutralizar o efeito do cátion dispersante $\left(\mathrm{Na}^{+}\right)$do solo. Com isto, sugere-se que o corretivo contenha principalmente cálcio $\left(\mathrm{Ca}^{2+}\right)$, para que este substitua o $\mathrm{Na}^{+}$no complexo de troca do solo. O gesso agrícola é um corretivo químico de uso freqüente na recuperação de solos com problemas de sódio trocável devido, principalmente, aos fatores: disponibilidade, economicidade e eficiência técnica (Richards, 1974).

Por se tratar de um sal neutro, o gesso se dissocia, quando em solução, formando $\mathrm{Ca}^{2+}$ e $\mathrm{SO}_{4}^{2-}$ e, deste modo, reage na solução do solo. A reação de troca de cátions, mais importante envolvendo gesso, ocorre justamente nos solos alcalinos ou sódicos, devido ao íon $\mathrm{Ca}^{2+}$ ser mais fortemente adsorvido ao solo que o íon $\mathrm{Na}^{2+}$; assim, grande parte do sódio trocável tenderá para a solução do solo (Raij, 1992). O íon sulfato tende a neutralizar o sódio em solução, originando o sulfato de sódio decaidratado $\left(\mathrm{Na}_{2} \mathrm{SO}_{4} \cdot 10 \mathrm{H}_{2} \mathrm{O}\right)$ que, após a adição de água, é lixiviado para as camadas mais profundas do perfil do solo (Santos, 1995).

Por outro lado, o uso de materiais orgânicos em suas diversas formas (esterco, adubação verde, palha de arroz), objetivando a recuperação de áreas com problemas de sódio trocável, também tem sido uma prática de relativa eficiência (Silva, 1978). Pesquisas mais recente (Rossiello et al., 1981; Ruiz et al., 1997; Silveira, 1999) têm sugerido a vinhaça (subproduto da cana-de-açúcar) como um corretivo promissor para uso na correção de solos com predominância de sódio trocável. Nessas áreas, em que no complexo de troca predomina o $\mathrm{Na}^{+}$trocável, além de condicionadores ou melhoradores químicos, a exemplo do gesso agrícola, tem sido recomendada a incorporação de materiais orgânicos, tais como: esterco de curral, adubo verde, composto de lixo urbano e resíduo de destilarias para a melhoria da permeabilidade desses solos (Silva, 1978). Holanda et al. (1998) constataram que o esterco de curral foi benéfico na redução do sódio trocável de um solo salino-sódico, principalmente quando associado à subsolagem e à gessagem.

Almeida (1994) estudando o efeito de torta de filtro e vinhaça como alternativas de recuperação de um solo com problemas de sódio trocável, constatou que o gesso apresentou melhores resultados, quando comparado com a torta de filtro e observou ainda que, a lixiviação do solo com vinhaça superou positivamente os resultados, provenientes da lixiviação com a água das chuvas, em todos os tratamentos. Silveira (1999) também constatou efeito positivo da vinhaça sobre as características produtivas de um solo sódico tratado com gesso, incubado com vinhaça e com água destilada. Resultados idênticos foram obtidos por Almeida (1994) e por Ruiz et al. (1997) utilizando vinhaça e água de chuva na lixiviação de um solo salino-sódico, vinhaça e água de chuva. Santos (1981) mostrou, em casa-de-vegetação, que a incubação da camada superficial de um Podzólico com dosagens crescentes de vinhaça (até $1.600 \mathrm{~m}^{3} \mathrm{ha}^{-1}$ ), induziu aumentos consistentes na condutividade elétrica do extrato de saturação do solo.

Com este trabalho objetivou-se estudar o uso de gesso agrícola e de composto de lixo urbano, além de verificar o período de incubação do solo com vinhaça, visando à recuperação de um solo com problemas de sódio trocável do perímetro irrigado São Gonçalo, no município de Sousa, PB, usando-se o algodoeiro herbáceo (Gossypium hirsutum L.r. latifolium Hutch) como cultura teste.

\section{MATERIAL E MÉTODOS}

O experimento foi conduzido no período de março a julho de 2001, em um galpão telado, coberto com telhas de fibra de vidro transparente, pertencente ao Centro de Ciências Agrárias da Universidade Federal da Paraíba (CCA/UFPB), localizado no município de Areia, PB. Usou-se o material de um solo com problemas de sódio trocável (Cordeiro \& Millar, 1978), 
Tabela 1. Resultado das análises física, química e de salinidade do material do solo $(0-20 \mathrm{~cm})$, da área do Setor 07 do Perímetro de Irrigação São Gonçalo, usado no trabalho

\begin{tabular}{|c|c|c|c|c|c|}
\hline \multicolumn{2}{|c|}{ Atributos Físicos } & \multicolumn{2}{|c|}{ Atributos Químicos } & \multicolumn{2}{|c|}{ Atributos Salinos } \\
\hline Areia, $\mathrm{g} \mathrm{kg}^{-1}$ & 594 & $\mathrm{pH}_{\mathrm{H} 2 \mathrm{O}}(1: 2,5)$ & 10,60 & $\mathrm{pH}$ & 9,82 \\
\hline Silte, $\mathrm{g} \mathrm{kg}^{-1}$ & 250 & $\mathrm{Na}\left(\mathrm{cmol}_{\mathrm{c}} \mathrm{dm}^{-3}\right)$ & 11,67 & $\mathrm{CE}^{3}\left(\mathrm{dS} \mathrm{m}^{-1}\right)$ & 3,80 \\
\hline Densidade, $\mathrm{kg} \mathrm{dm}^{-3}$ & & $\mathrm{Mg}\left(\mathrm{cmol}_{\mathrm{c}} \mathrm{dm}^{-3}\right)$ & 0,30 & $\mathrm{Ca}\left(\mathrm{mmol}_{\mathrm{c}} \mathrm{L}^{-1}\right)$ & 2,00 \\
\hline Solo & 1,79 & $\mathrm{~K}\left(\mathrm{mg} \mathrm{dm}^{-3}\right)$ & 60,00 & $\operatorname{Mg}\left(\mathrm{mmol}_{\mathrm{c}} \mathrm{L}^{-1}\right)$ & 0,50 \\
\hline Partículas & 2,80 & $\mathrm{H}+\mathrm{Al}\left(\mathrm{cmol}_{\mathrm{c}} \mathrm{dm}^{-3}\right)$ & 0,00 & $\mathrm{~K}\left(\mathrm{mmol}_{\mathrm{c}} \mathrm{L}^{-1}\right)$ & 0,14 \\
\hline $1,50 \mathrm{MPa}$ & 6,61 & $\mathrm{M} \mathrm{O}^{2}\left(\mathrm{~g} \mathrm{~kg}^{-1}\right)$ & 1,97 & & \\
\hline
\end{tabular}

proveniente de um solo aluvial, classificado como Neossolo Flúvico (EMBRAPA, 1999), coletado na profundidade de 0-20 $\mathrm{cm}$ do perfil da área do Setor 7 do Perímetro Irrigado São Gonçalo, conduzido ao laboratório e submetido às análises física, química e de salinidade, segundo metodologia da EMBRAPA (1997), cujos resultados se encontram na Tabela 1.

Os tratamentos foram constituídos por duas dosagens de gesso agrícola, 0 e $40 \%$ da necessidade de gesso $(\mathrm{NG}=14,5 \mathrm{~g}$ $\mathrm{kg}^{-1}$ ), segundo determinação de Richards (1974), duas de composto de lixo urbano (CLU), 0 e $20 \mathrm{t} \mathrm{ha}^{-1}$ e quatro períodos de incubação do solo com vinhaça: 15, 30, 45 e 60 dias. Para avaliar o possível nível de recuperação do solo, usou-se o algodoeiro herbáceo, cultivar CNPA-7H, durante os primeiros 35 dias do ciclo da cultura. A vinhaça apresentou, como características químicas, $\mathrm{pH}=3,5 ; \mathrm{CE}=6,7 \mathrm{dS} \mathrm{m}^{-1}$; matéria seca $=4,25 \% ; \mathrm{N}=0,21 \mathrm{~g} \mathrm{~L}^{-1} ; \mathrm{P}=0,21 \mathrm{~g} \mathrm{~L}^{-1} ; \mathrm{Ca}=0,76 \mathrm{~g} \mathrm{~L}^{-1}$; $\mathrm{Mg}=0,54 \mathrm{~g} \mathrm{~L}^{-1} \mathrm{e} \mathrm{K}=1,69 \mathrm{~g} \mathrm{~L}^{-1}$.

Adotou-se o delineamento experimental em blocos ao acaso com os tratamentos distribuídos em arranjo fatorial 2 × 2 × 4 (duas dosagens de gesso, duas de composto de lixo urbano e quatro períodos de incubação), com três repetições. A unidade experimental foi representada por um vaso plástico com capacidade para $2,5 \mathrm{dm}^{3}$ contendo $2 \mathrm{~kg}$ de solo secado ao ar.

A implantação do ensaio ocorreu em 9/3/2001. Após as pesagens do gesso e da matéria orgânica, esses materiais foram devidamente misturados ao solo e, em seguida, incubados com $300 \mathrm{~mL}$ de vinhaça (aproximadamente metade da saturação do solo). Os períodos de incubação do solo foram de $9 / 3$ a 23/3/ 2001, $9 / 3$ a $7 / 4 / 2001,9 / 3$ a $22 / 4 / 2001$ e de $9 / 3$ a $7 / 5 / 2001$ correspondendo, respectivamente, a 15, 30, 45 e 60 dias. Durante a incubação os tratamentos permaneceram em condições de umidade próximas à capacidade de campo, com aplicação de água proveniente do açude de São Gonçalo.

Após os períodos de incubação, os tratamentos foram submetidos a lavagem dos sais solúveis do solo, utilizando-se água do açude de São Gonçalo classificada, do ponto de vista de salinidade/sodicidade, como $\mathrm{C}_{1} \mathrm{~S}_{1}$, conforme Richards (1974), adicionada de maneira intermitente, por ser esta forma considerada mais eficiente que a lixiviação contínua (Ayers \& Westcot, 1991). Nesta prática colocou-se igual volume de água em cada vaso e, após a drenagem, o processo se repetiu até que a condutividade elétrica do lixiviado final atingisse condições de estabilidade. Em média, foram colocados 2000 $\mathrm{mL}$ de água por tratamento, para drenar.
Nos lixiviados foram determinados $\mathrm{pH}$, condutividade elétrica (CE), cátions sódio e cálcio $\left(\mathrm{Na}^{+} \mathrm{e} \mathrm{Ca}^{2+}\right)$; para o desenvolvimento da cultura foram avaliadas a percentagem de emergência (\%E) e a produção de biomassa seca (BS) da parte aérea. Os resultados foram submetidos a análise de variância (teste F), com desdobramento dos efeitos das interações significativas, com as médias comparadas, entre si, pelo teste de Tukey, a 5\% de probabilidade (Gomes, 1990). Para os resultados dos períodos de incubação, foi procedida a análise de regressão polinomial, em que a escolha do modelo foi para o maior grau do efeito significativo.

\section{RESULTADOS E DISCUSSÃO}

A Tabela 2 revela efeito dos tratamentos $(\mathrm{p} \leq 0,01)$ sobre os resultados do sódio nos lixiviados, enquanto o cálcio, o pH e a produção de biomassa seca (BS) do algodão $\mathrm{CNPA}-7 \mathrm{H}$, foram afetados apenas pelo gesso e os períodos de incubação do solo com vinhaça. Para o $\mathrm{Ca}^{2+}$ ocorreu interação para todas as combinações possíveis, ao passo que para a condutividade elétrica (CE), $\mathrm{Na}^{+}$, percentagem de emergência (\%E) e BS, a interação foi apenas para necessidade de gesso versus períodos de incubação.

Através da comparação entre as médias pelo teste de Tukey a 5\% de probabilidade (Tabela 3) é possível afirmar que a aplicação do gesso e do CLU, isolado ou associado, concorreu para reduzir, de forma significativa ( $\mathrm{p} \leq 0,05)$ os resultados do pH nos lixiviados, em relação ao solo incubado com vinhaça, tendo o gesso causado redução mais expressiva que o CLU. Em geral, houve redução no $\mathrm{pH}$ dos lixiviados entre os tratamentos, da ordem de 13,1; 15,8 e 18,3\% para a vinhaça, CLU e gesso, respectivamente, em relação ao $\mathrm{pH}(9,82)$ do extrato de saturação do solo, antes dos tratamentos. Almeida (1994), em um solo com elevado teor de sódio trocável e $\mathrm{pH}=11,00$ do extrato de saturação, submetido a lixiviação com vinhaça, observou redução do $\mathrm{pH}$ do lixiviado para 6,08. Idêntica redução também se verificou quando o solo foi tratado com gesso mais torta de filtro. Ruiz et al. (1995) constataram que o $\mathrm{pH}$ dos lixiviados de um solo sódico decresceu com os tratamentos nos quais se utilizou gesso, torta de filtro, gesso + torta de filtro e vinhaça, em 10,4, 11,3, 18,9 e 39,6\%, respectivamente, em relação ao $\mathrm{pH}$ do extrato do solo $(10,6)$, antes dos tratamentos. 
Tabela 2. Resumo de análise de variância referentes ao $\mathrm{pH}$, condutividade elétrica $(\mathrm{CE})$, cátions $\mathrm{Na}^{+}$e $\mathrm{Ca}^{2+}$, percentagem de emergência (\%E) e biomassa seca da parte aérea (BS), do algodão CNPA-7H, em função dos tratamentos de gesso, composto de lixo urbano e período de incubação do solo com vinhaça

\begin{tabular}{|c|c|c|c|c|c|c|c|}
\hline \multirow[b]{2}{*}{ C. da variação } & \multirow[b]{2}{*}{ GL } & \multicolumn{6}{|c|}{ Quadrado Médio } \\
\hline & & $\mathrm{pH}$ & $\mathrm{CE}$ & $\mathrm{Na}^{+}$ & $\mathrm{Ca}^{2+}$ & $\% \mathrm{E}$ & $\mathrm{BS}$ \\
\hline NG & 1 & $1,59^{* *}$ & $0,32^{\mathrm{ns}}$ & $1114,7 * *$ & $2336,6^{* *}$ & $1692,2^{\text {ns }}$ & $0,32^{* *}$ \\
\hline CLU & 1 & $0,17^{\text {ns }}$ & $0,58^{\mathrm{ns}}$ & $209,6^{*}$ & $22,5^{\mathrm{ns}}$ & $150,6^{\mathrm{ns}}$ & $0,08^{\mathrm{ns}}$ \\
\hline Período (P) & 3 & $0,61 * *$ & $1,09^{\mathrm{ns}}$ & $1314,7 * *$ & $1171,0 * *$ & $260,2^{\text {ns }}$ & $0,04^{* *}$ \\
\hline NG vs CLU & 1 & $0,22^{\mathrm{nn}}$ & $0,07^{\mathrm{ns}}$ & $115,0^{\mathrm{ns}}$ & $129,7 *$ & $88,0^{\text {ns }}$ & $0,03^{\mathrm{ns}}$ \\
\hline NG vs $P$ & 3 & $0,18^{\mathrm{ns}}$ & $2,67^{* *}$ & $321,1 * *$ & $599,2 * *$ & $647,7 * *$ & $0,07^{* *}$ \\
\hline CLU x P & 3 & $0,27^{\mathrm{ns}}$ & $1,08^{\mathrm{ns}}$ & $90,2^{\mathrm{ns}}$ & $75,0 *$ & $22,7^{\text {ns }}$ & $0,03^{\mathrm{ns}}$ \\
\hline NG vs CLU vs $P$ & 3 & $0,07^{\mathrm{ns}}$ & $0,20^{\mathrm{ns}}$ & $56,5^{\text {ns }}$ & $125,7 * *$ & $299,1^{\mathrm{ns}}$ & $0,08^{\mathrm{ns}}$ \\
\hline Linear & 1 & $0,97^{* *}$ & $5,81^{* *}$ & $10,3^{\mathrm{ns}}$ & $190,8^{* *}$ & $1260,4^{* *}$ & $0,06^{* *}$ \\
\hline Quadrática & 1 & $0,75^{*}$ & $0,234^{\mathrm{ns}}$ & $92,4^{\mathrm{ns}}$ & $0,7^{\mathrm{ns}}$ & $602,1^{*}$ & $0,01^{\mathrm{ns}}$ \\
\hline Cúbica & 1 & $0,45^{\mathrm{ns}}$ & $0,18^{\text {ns }}$ & $177,8^{\text {ns }}$ & $7,4^{\mathrm{ns}}$ & $93,6^{\mathrm{ns}}$ & $0,01^{\mathrm{ns}}$ \\
\hline (Tratamentos) & 15 & 0,36 & 1,07 & 472,5 & 560,0 & 394,7 & 0,05 \\
\hline Blocos & 2 & 0,09 & 3,75 & $55,7^{\text {ns }}$ & $39,7^{\mathrm{ns}}$ & 484,9 & 0,02 \\
\hline Resíduos & 30 & 0,109 & 0,59 & 42,2 & 19,0 & 111,6 & 0,007 \\
\hline
\end{tabular}

Tabela 3. Resultados médios do $\mathrm{pH}$, condutividade elétrica (CE), sódio $\left(\mathrm{Na}^{+}\right)$, cálcio $\left(\mathrm{Ca}^{2+}\right)$ percentagem de emergência e de biomassa seca da parte aérea do algodão CNPA-7H, em função das dosagens de gesso (NG), composto de lixo urbano (CLU) e dos períodos de incubação de um solo com problema de sódio trocável

\begin{tabular}{ccccccc}
\hline Tratamento NG:CLU & $\mathrm{pH}$ & $\mathrm{CE}\left(\mathrm{dS} \mathrm{m}^{-1}\right)$ & $\mathrm{Na}^{+}\left(\mathrm{mmol}_{\mathrm{c}} \mathrm{L}^{-1}\right)$ & $\mathrm{Ca}^{2+}\left(\mathrm{mmol}_{\mathrm{c}} \mathrm{L}^{-1}\right)$ & Emergência (\%) & Biomassa (mg) \\
\hline $0: 00$ & $8,53 \mathrm{a}$ & $2,59 \mathrm{a}$ & $68,69 \mathrm{a}$ & $16,75 \mathrm{~b}$ & $76,0 \mathrm{c}$ & $420,5 \mathrm{c}$ \\
$0: 20$ & $8,27 \mathrm{~b}$ & $2,89 \mathrm{a}$ & $67,61 \mathrm{a}$ & $12,09 \mathrm{c}$ & $80,2 \mathrm{bc}$ & $534,2 \mathrm{~b}$ \\
$40: 0$ & $8,02 \mathrm{c}$ & $2,50 \mathrm{a}$ & $62,15 \mathrm{~b}$ & $27,41 \mathrm{a}$ & $83,7 \mathrm{ab}$ & $672,5 \mathrm{a}$ \\
$40: 20$ & $8,04 \mathrm{c}$ & $2,64 \mathrm{a}$ & $54,93 \mathrm{c}$ & $29,37 \mathrm{a}$ & $87,9 \mathrm{a}$ & $714,2 \mathrm{a}$ \\
Média & 8,22 & 2,66 & 63,33 & 21,40 & 822,0 & 611,9 \\
CV\% & 4,01 & 18,92 & 10,25 & 20,37 & 12,9 & 13,3 \\
dms/NG/CLU & 0,19 & - & 3,83 & 2,57 & 6,2 & 48,0 \\
\hline
\end{tabular}

NG: \% da necessidade de gesso; CLU: Composto de lixo urbano ( $\left.\mathrm{t} \mathrm{ha}^{-1}\right)$; CV: Coeficiente de variação (\%); dms: Diferença mínima significativa Médias seguidas de mesma letra na vertical não diferem significativamente entre si pelo teste de Tukey, a $5 \%$ de probabilidade

Ainda nos resultados da Tabela 3, constata-se ausência de efeito dos tratamentos sobre os resultados da $\mathrm{CE}$, porém em média essa variável foi reduzida em $30 \%$ em relação à $\mathrm{CE}$ do extrato de saturação do solo, antes de receber os tratamentos (Tabela 1). Almeida (1994) em um solo com problemas de sódio trocável, submetido a lixiviação com vinhaça, também obteve redução na CE dos lixiviados em $17,2 \%$, quando o solo foi tratado com gesso em relação à condutividade elétrica do extrato da saturação do solo. Ruiz et al. (1995) também verificaram decréscimos na $\mathrm{CE}$ dos lixiviados de um solo sódico tratado com torta de filtro, gesso, gesso + torta de filtro e vinhaça, da ordem de 79,2, 85,3, 90,4 e 92,5\%, respectivamente, em relação à $\mathrm{CE}\left(37,5 \mathrm{dS} \mathrm{m}^{-1}\right)$ do extrato do solo antes dos tratamentos.

$\mathrm{O}$ gesso, isolado ou associado ao CLU, concorreu para reduzir, de forma significativa, os teores de sódio nos lixiviados, não tendo ocorrido diferença entre os resultados da vinhaça e do CLU. Situação inversa se deu com o cálcio, em que sua menor concentração $\left(16,75 \mathrm{cmol}_{c} \mathrm{~L}^{-1}\right)$ se verificou no solo tratado apenas com vinhaça, ou seja, na ausência do gesso e do CLU. Almeida (1994) constatou, em solo com problema de sódio trocável tratado com gesso e drenado com vinhaça, que o sódio no lixiviado foi reduzido com o tempo de drenagem, fato este atribuído ao efeito da vinhaça, em razão da grande quantidade de $\mathrm{Na}^{+}$solúvel encontrada nos primeiros dias. Misopolinos
(1985), também constatou redução do sódio nos lixiviados na correção de solos sódicos. Para Cavalcante \& Silveira (1985), um solo salino sódico tratado com fosfogesso contribuiu para

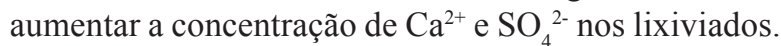

Constatou-se, através dos dados da Tabela 3, que a aplicação do CLU em comparação com o gesso, aumentou de forma significativa $(\mathrm{p} \leq 0,05)$ os teores de $\mathrm{Na}^{+}$, mas reduziu os de $\mathrm{Ca}^{2+}$ nos lixiviados. Não se verificou diferença entre o CLU e a vinhaça em relação aos teores de sódio nos lixiviados, mas para o cálcio a vinhaça superou significativamente o CLU. Ao se fazer uma apreciação quanto aos efeitos dos tratamentos de gesso e do CLU, isolados ou associados, deve-se enfatizar a participação da vinhaça no processo, visto este produto conter, em sua constituição, quantidade apreciável de cálcio $(0,76 \mathrm{~g}$ de $\left.\mathrm{Ca}^{2+} \mathrm{L}^{-1}\right)$ que, de certa forma, contribuiu para o aumento do sódio nos lixiviados, decorrente do efeito de substituição do $\mathrm{Ca}^{2+}$ pelo $\mathrm{Na}^{+}$no complexo de troca.

Para os resultados médios de emergência das plântulas $(\% \mathrm{E})$ e produção de biomassa seca da parte aérea (BS), é possível se constatar que a presença do gesso, isolado ou associado ao CLU favoreceu, de forma significativa, o estabelecimento da cultura, avaliado em termos da \%E e da BS (Tabela 3); sem dúvida, importante parcela do $\mathrm{Na}^{+}$no complexo de troca deve ter sido substituída pelo $\mathrm{Ca}^{2+}$ do gesso reduzindo, desta forma, 
o efeito do sódio e melhorando algumas características produtivas do solo, como por exemplo o sódio trocável. A percentagem de emergência das plântulas e a biomassa seca da parte aérea aumentaram em ordem significativa com a aplicação do CLU e do gesso, em relação ao solo tratado com vinhaça.

Os resultados do $\mathrm{pH}$ dos lixiviados submetidos a análise de regressão polinomial para os tratamentos de NG e de CLU, em função dos períodos de incubação do solo com vinhaça, revelaram que não houve efeito de período de incubação sobre o pH dos lixiviados para os tratamentos isolados da $\mathrm{NG}$ e do CLU mas, sim, para a mistura de NG + CLU. De acordo com a Figura 1, o efeito de período de incubação sobre o $\mathrm{pH}$ do lixiviado do tratamento NG + CLU foi de natureza quadrática, cujo modelo obtido permite que se afirme que o valor máximo da função $(\mathrm{pH}=8,17)$ seria atingido, teoricamente, com um período de incubação de 52 dias, aproximados. Esta informação é indicativa de que o solo tratado com gesso e com composto de lixo urbano necessitou de maior período para o equilíbrio das reações no complexo solo-solução no processo de estabilização dos eletrólitos. Mesmo não estudando o período de incubação de um solo com problemas de sódio trocável mas, sim, o tempo de lixiviação com vinhaça (Almeida, 1994), constatou que o $\mathrm{pH}$ do lixiviado decresceu com o tempo de drenagem (20 dias) para o solo tratado com gesso e/ou torta de filtro.

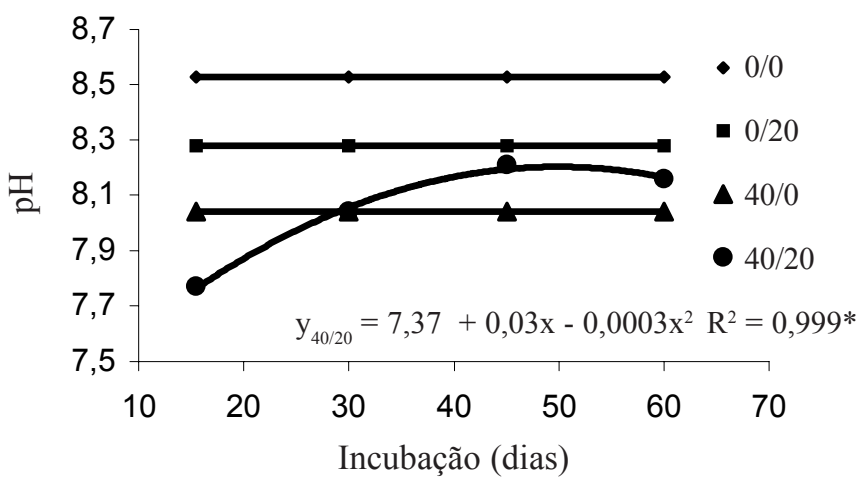

Figura 1. Resultados do $\mathrm{pH}$ dos lixiviados em função dos períodos de incubação $(15,30,45$ e 60 dias $)$ do solo com vinhaça para os tratamentos (NG:CLU): 0:0, 0:20, 40:0 e 40:20

Para a condutividade elétrica dos lixiviados $(\mathrm{CE})$, porém os resultados dos tratamentos 0:0 e 40:2 revelaram, a partir da regressão polinomial efeito de natureza quadrática, cujos modelos permitem afirmar-se que, para o caso do tratamento testemunha (0:0) a CE atingiria um máximo $\left(3,2 \mathrm{dS} \mathrm{m}^{-1}\right)$, teoricamente para um período de incubação de 64,3 dias, enquanto para o tratamento 40:20 o modelo obtido permitiu um mínimo de 47,5 dias (Figura 2). Certamente, a incubação do solo com vinhaça deva ter concorrido para que a CE do tratamento testemunha crescesse com o período de incubação, até atingir um máximo para, em seguida, apresentar tendência de decréscimo, conforme o modelo ajustado aos dados obtidos e também para que a CE do tratamento $40 / 20$ decrescesse com o aumento do período de incubação, até alcançar um mínimo, para então se comportar com tendência de crescimento. Para

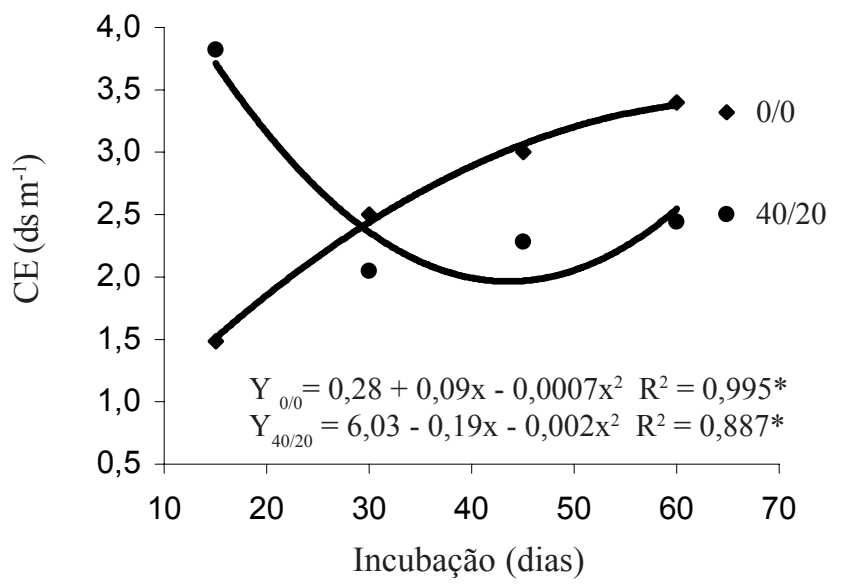

Figura 2. Resultados médios da condutividade elétrica (CE) dos lixiviados em função dos períodos de incubação $(15,30$, 45 e 60 dias) do solo com vinhaça, para os tratamentos (NG:CLU): 0:0 e 40:20

os tratamentos 0:20 e 40:0 não houve efeito dos períodos de incubação sobre os resultados da condutividade elétrica (CE) dos lixiviados. Segundo Almeida (1994) a condutividade elétrica dos lixiviados decresce com o período de drenagem do solo com vinhaça.

A análise de regressão polinomial não revelou efeito dos períodos de incubação sobre os resultados do sódio nos lixiviados para o tratamento testemunha (0:0). Conforme Fig. 3, ocorreu efeito linear decrescente dos períodos de incubação para os tratamentos 0:20, 40:0 e 40:20 sobre os resultados de sódio nos lixiviados. Segundo os modelos obtidos, os decréscimos foram de 0,$54 ; 0,76$ e $0,79 \mathrm{mmol} \mathrm{de} \mathrm{Na}^{+} \mathrm{dia}^{-1}$, para os tratamentos 0:20, 40:0 e 40:20, respectivamente. Na mesma ordem e de acordo com os coeficientes de determinação obtidos, os resultados foram explicados em 99,90 e $98 \%$ pelo efeito dos tratamentos de período de incubação. Comportamento semelhante também foi constatado por Almeida (1994), independentemente do tratamento, se na ausência ou na presença de gesso e de torta de filtro, isolados ou associados; o sódio decresceu nos lixiviados com o período de drenagem do solo com vinhaça. Pratter et al. (1978), avaliando a eficiência de vários produtos na correção de solos sódicos, constataram que a quantidade de sódio nos lixiviados, em valores acumulados, aumentou no decorrer do tempo, principalmente quando o condicionador utilizado foi o gesso. Para Ruiz et al. (1995), a quantidade de sódio nos lixiviados aumentou, respectivamente, com o uso do gesso, gesso + torta de filtro, torta de filtro e vinhaça.

Para os resultados de cálcio nos lixiviados, a análise de regressão revelou efeito linear para os tratamentos 0:0 e 40:20 com decréscimo de $0,24 \mathrm{e} 0,91 \mathrm{mmol}_{\mathrm{c}} \mathrm{Ca} \mathrm{dia}^{-1}$, respectivamente, e efeito quadrático para o tratamento 40:0, cujo modelo estimado apresentou um mínimo de 13,5 mmoles de $\mathrm{Ca}^{2+}$ aos 49,5 dias (Figura 4). Para o tratamento 0:20 a análise de regressão não comprovou efeito dos períodos de incubação sobre os resultados de $\mathrm{Ca}^{2+}$.

De acordo com a análise de regressão, não se constatou efeito dos períodos de incubação sobre os resultados de emergência das plântulas para o tratamento 40:20. Pela Figura 5 , é possível se afirmar, em relação aos tratamentos 0:0, 0:20 e 


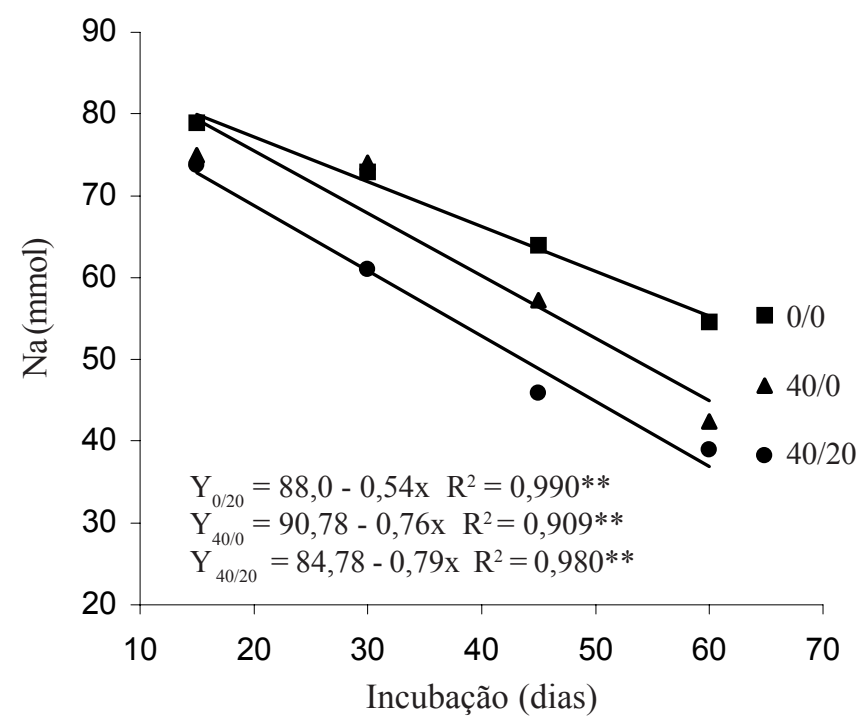

Figura 3. Resultados médios do sódio dos lixiviados em função dos períodos de incubação (15, 30, 45 e 60 dias) do solo com vinhaça, para os tratamentos (NG:CLU): 0:20, 40:0 e 40:20

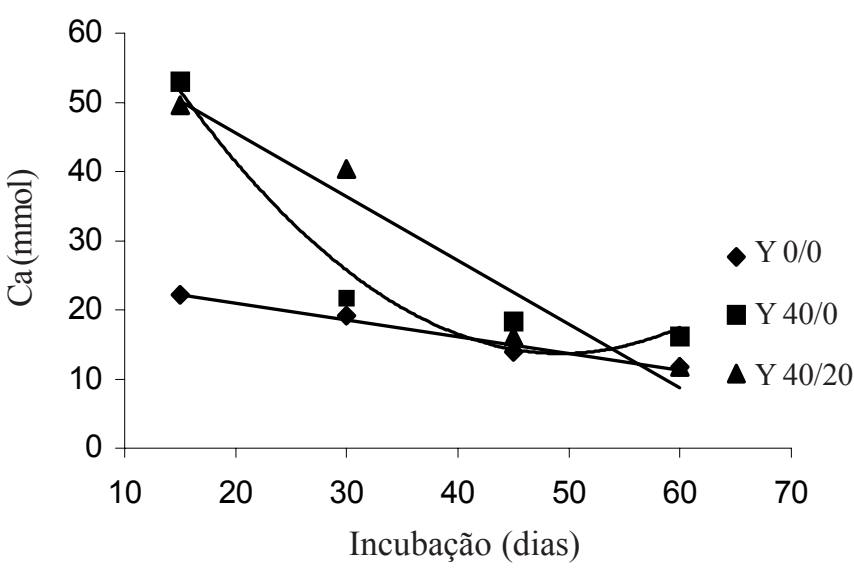

Figura 4. Resultados médios do cálcio nos lixiviados em função dos períodos de incubação (15, 30, 45 e 60 dias) do solo com vinhaça, para os tratamentos (NG:CLU): 0:0, 40:0 e 40:20

40:0, efeito do $2^{\circ}$ grau dos períodos de incubação sobre os resultados da \%E das plântulas. Conforme os modelos obtidos teoricamente, os períodos de incubação proporcionariam aos 19, 33 e 69 dias, os valores máximos de 86, 77 e 100\% de emergência das plântulas, respectivamente, para os tratamentos 0:0, 0:20 e 40:0. Os resultados sinalizam que para o solo sódico em processo de recuperação, tratado com gesso, composto de lixo urbano e/ou incubado com vinhaça, deve ser observado o período de incubação para que o cálcio nos lixiviados seja reduzido, dando oportunidade para que esse cátion substitua o $\mathrm{Na}^{+}$no complexo de troca, eliminando o efeito pernicioso do sódio trocável no solo.

Evidenciou-se, através da análise de regressão, efeito dos períodos de incubação do solo com vinhaça sobre os resultados da produção de biomassa seca da parte aérea, apenas para o tratamento testemunha (0:0), cujo efeito foi de natureza linear decrescente (Figura 6) e onde, segundo o modelo estimado, teoricamente ocorreu decréscimo da ordem de 6,3 $\mathrm{mg}$ de matéria seca para cada dia de incubação.

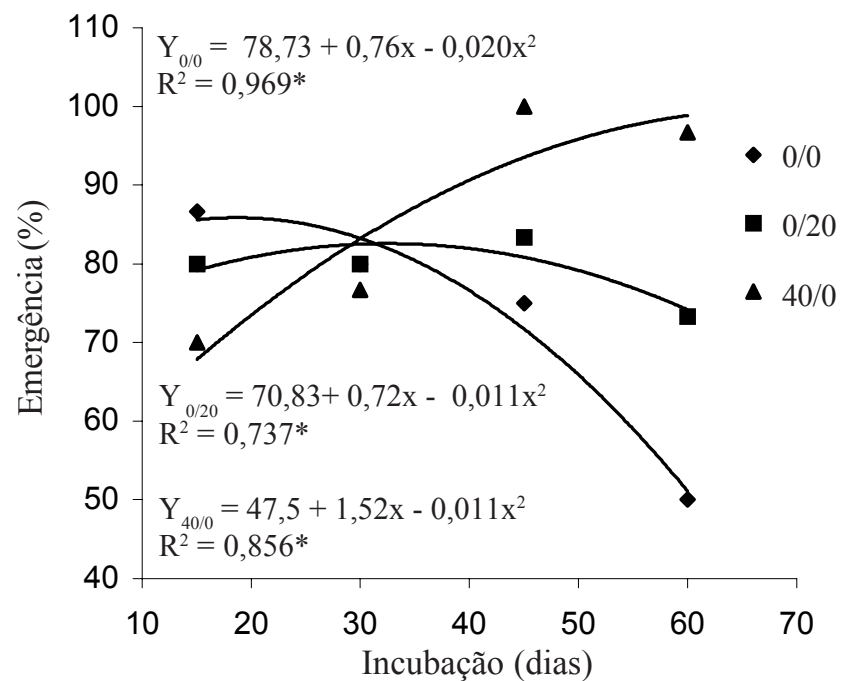

Figura 5. Resultados médios referentes à emergência das plântulas em função dos períodos de incubação (15, 30, 45 e 60 dias) do solo com vinhaça para os tratamentos (NG:CLU): 0:0, 0:20 e 40:0

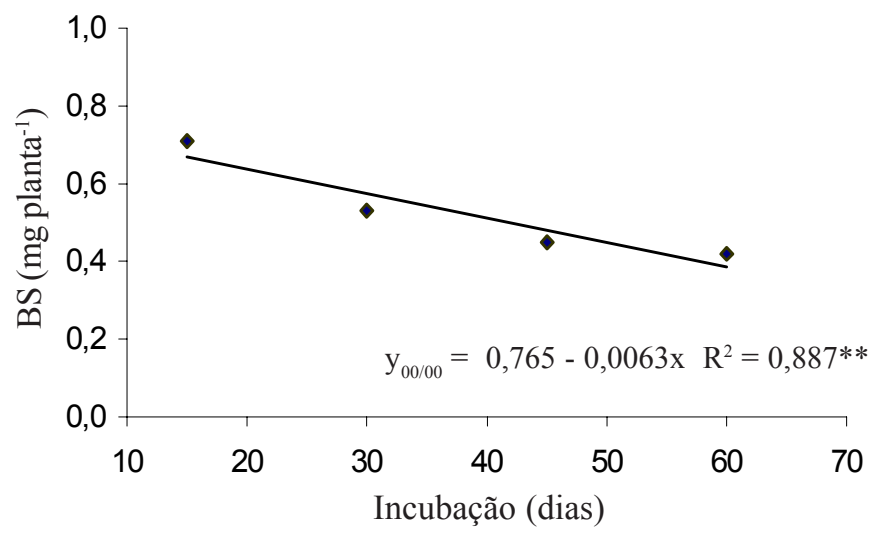

Figura 6. Resultados médios referentes à biomassa seca (BS) da parte aérea das plântulas em função dos períodos de incubação ( $\mathrm{P}_{1} \mathrm{P}_{2} \mathrm{P}_{3}, \mathrm{P}_{4}=15,30,45$ e 60 dias, respectivamente) do solo com vinhaça, para os tratamentos NG:CLU (0:0)

\section{CONCLUSÕES}

1. O gesso, isolado ou associado ao CLU, reduziu o $\mathrm{pH}$ e os teores de $\mathrm{Na}^{+}$nos lixiviados, porém aumentou os de $\mathrm{Ca}^{2+}$, a emergência das plântulas e a biomassa seca.

2. O composto de lixo urbano reduziu o $\mathrm{pH}$ e o $\mathrm{Ca}^{2+}$, mas aumentou a produção de biomassa seca.

3. A vinhaça, isolada ou associada ao CLU, foi menos eficiente na redução do pH e dos teores de sódio nos lixiviados.

4. Os períodos de incubação do solo apresentaram efeitos diferenciados sobre as variáveis analisadas, segundo os tratamentos de gesso, CLU e vinhaça, isolados ou associados.

\section{LITERATURA CITADA}

Almeida, M.T. Torta de filtro e vinhaça como alternativas na recuperação de um solo salino-sódico. Viçosa: UFV, 1994. 52p. Dissertação Mestrado 
Ayers, R.S.; Westcot, D.V.A. A qualidade da água na agricultura. Campina Grande, PB: 1991. 2218p. Estudos FAO: Irrigação e Drenagem, 29 Revisado

Bernardo, S. Manual de irrigação. 6 ed. Viçosa: Imprensa Universitária, UFV. 1995. 657p.

Cavalcante, L.F.; Silveira, R.I. Importância do fosfogesso como fonte de cálcio, enxofre e na redução de sódio de um solo salino-sódico da Paraíba. In: Congresso Brasileiro de Ciência do Solo, 10, Belém. Anais..., Belém: SBCS/UFPA/ EMBRAPA, 1985, p.118.

Christofidis, D. Prática de irrigação no mundo. Item: Brasilia, n.49, p.8-13.2001.

Cordeiro, G.G.; Barreto, A.N.; Carvalho, A.C.N. Levantamento das condições de salinidade e sodicidade do Projeto de Irrigação de São Gonçalo ( $2^{a}$ parte). Petrolina: EMBRAPA/ CPATSA, 1988. 57p. Documento 54

Cordeiro, G.G.; Millar, A.M. Caracterização dos problemas de sais dos setores de irrigação em operação do Projeto São Gonçalo. In: Reunião sobre salinidade em áreas irrigadas, Fortaleza, 1978, Anais..., Recife: SUDENE, 1978, p.25-34.

Damasceno, J.H. Informe de drenagem e salinidade nos perímetros irrigados do Departamento Nacional de Obras Contra as Secas. In: Reunião sobre salinidade em áreas irrigadas, Fortaleza, 1978. Anais... Fortaleza: DNOCS, 1978, p.113-123.

EMBRAPA. Centro Nacional de Pesquisa de Solos. Manual de métodos de análise de solo 2. ed. Rio de Janeiro, 1997, 212p. Embrapa - CNPS, Documento 1

EMBRAPA. Centro Nacional de Pesquisa de Solos (Rio de Janeiro). Sistema brasileiro de classificação de solos, Brasília: Embrapa, p.1. 1999. 412p.

Góes, E.S. de. O problema da salinidade e drenagem em projetos de irrigação no Nordeste e a ação da pesquisa com vistas ao seu equacionamento. Recife: SUDENE, 1978.20p.

Gomes, P.F. Curso de estatística experimental. Piracicaba: Nobel, $1990.468 \mathrm{p}$

Gupta, R.K.; Abrol, I.P. Salt-affected soils: their reclamation and management for crop production. Advances in Soil Sciences, New York, v.11, p.224-288, 1990.

Holanda, J.S.; Vitti, G.C.; Salviano, A.A.C.; Medeiros, J.D.F.; Amorim, J.R.A. Alterações nas propriedades químicas de um solo aluvial salino-sódico decorrentes da subsolagem e do uso de condicionadores. Revista Brasileira de Ciência do Solo, Viçosa, v.22, n.3, p.387-394. 1998.

Misopolinos, N.D. A new concept for reclaiming sodic soils with high-salt water. Soil Science, Baltimore, v.140, n.1, p.6974, 1985 .
Pratter, R.J.; Goertzen, J.O.; Rhoades, J.D.; Frenkel, H. Efficient amendment use in sodic soil reclamation. Soil Science Society of America Journal, Madison, v.42, n.5, p.782-86, 1978.

Rhoades, J.D.; Loveday, J. Salinity in irrigated agriculture. In: Stewart, D.R.; Nielson, D.R. (eds) Irrigation of agricultural crops. Madison: ASA/CSSA/SSSA, 1990. cap.36, p.10891142. Agronomy, 30

Richards, L.A. Diagnostico y rehabilitacion de suelos salinos y sódicos. México: Editorial Limusa, 1974, 172p.

Rossiello, R.O.; Ferreira, W.de A.; Fernandes, M.S. Resposta de milho (Zea mays L.) à salinidade do solo induzida por aplicações de vinhaça I. Produção de matéria seca e área foliar. Revista Brasileira de Ciência do Solo, Campinas, v.5, n.3, p.171-175, 1981.

Ruiz, H.A.; Gheyi, H.R.; Almeida, M.T.; Ribeiro, A.C. Torta de filtro e vinhaça como alternativas na recuperação de um solo salino-sódico.In: Congresso Brasileiro de Ciências do Solo, 25, Viçosa. Anais...., Viçosa: UFV/SBCS, 1995. p.20562268.

Ruiz, H.A.; Gheyi, H.R.; Almeida, M.T.; Ribeiro, A.C. Torta de filtro e vinhaça na recuperação de um solo salino-sódico e no desenvolvimento de arroz irrigado. Revista Brasileira de Ciência do Solo, Viçosa, v.21, n.4, p.659-665. 1997.

Santos, J.A.S. Efeito da temperatura, pré-embebição e salinidade na germinação e vigor de sementes de algodão (Gossypium hirstum L.) Campina Grande: UFPB/CCT, 1981. 91p. Dissertação Mestrado

Santos, J.G.R. dos. Desenvolvimento e produção da bananeira nanica sobre diferentes níveis de salinidade e lâminas de água. Campina Grande: UFPB. 173p. 1997. Tese Doutorado

Santos, R.V. dos. Correção de um solo salino-sódico e absorção de nutrientes pelo feijoeiro vigna (Vigna unguiculata (L) Walp). Piracicaba: ESALQ, 1995. 120p. Dissertação Mestrado

Silva, M.J. Efeito de diferentes métodos de recuperação num solo com problemas de sódio no Projeto de irrigação de São Gonçalo. PB. Viçosa: UFV, 1978. 54p. Dissertação Mestrado

Silveira, R.R. da. Recuperação de um solo afetado por sódio: Efeito do gesso e da incubação com vinhaça e água destilada. Areia: UFPB/CCA, 1999. 53p. Trabalho de Conclusão de Curso

SUDENE - Superintendência do desenvolvimento do Nordeste. Relatório Anual. Recife: DRN/SUDENE 1977. 82p.

Valdivieso, C.R. Salinidade de solos sob irrigação. In: Reunião Nacional para a Avaliação das Necessidades, Estabelecimento de Planejamento das Pesquisas Importantes para o Desenvolvimento dos Programas. Petrolina: EMBRAPA / CPATSA, 1986, 16p.

van Raij, V.B. Reações de gesso em solos ácidos. In: Seminário sobre o uso do gesso na agricultura, 2., Uberaba, 1992. São Paulo: IBRAFOS, 1992. p.106-119. 